\title{
Treatment of Hepatitis C in HIV-Infected Patients: Moving Towards an Era of All Oral Regimens
}

\author{
Ting-Yi Chen, MD, MPH, and Mamta K. Jain, MD, MPH
}

\begin{abstract}
Hepatitis C (HCV)-related liver disease has become one of the leading causes of death in HIV patients. With the development of new direct-acting antivirals for $\mathrm{HCV}$, treatment regimens have become shorter, more effective, and easier to tolerate without interferon. However, cost may be a significant impediment to the widespread use of these newer agents in both resource-rich and resource-poor settings. In HIV patients, treatment for HCV is not always as straightforward compared with HCV monoinfected patients due to potential drug-drug interactions. In this article, we will examine by genotypes the FDA approved direct-acting antivirals, as well as those in clinical trials that will soon be FDA-approved focusing on data in HCV/HIV co-infection. Preferred agents for HCV treatment and potential drug-drug interactions with antiretroviral therapy (ART) will be highlighted.
\end{abstract}

\section{Introduction}

A N ESTIMATED ONE-THIRD OF PATIENTS infected with HIV are co-infected with hepatitis C (HCV). Since 2007, the rate of death resulting from HCV has exceeded the rate of death due to HIV. ${ }^{1}$ In HIV-infected patients, life expectancy in those with HIV alone is approaching that of the general population, but not among those with HIV and HCV co-infection. In one study, the projected life expectancy in co-infected patients was approximately 20 years shorter than those with HIV alone, ${ }^{2}$ due in part to increasing rates of hepatocellular carcinoma (HCC). ${ }^{3}$ Historical rates of sustained viral response (SVR) with pegylated interferon (PegIFN) and ribavirin (RBV) therapy in genotype 1 were $29 \% .^{4}$ In addition, PegIFN had many side effects, and many patients with severe mental illness were excluded. RBV interacts with specific nucleoside reverse transcriptase inhibitors (NRTI) such as didanosine and stavudine, leading to a higher risk of lactic acidosis, and RBV-induced anemia can be exacerbated by zidovudine. ${ }^{5,6}$ Furthermore, the combination of PegIFN and RBV had to be administered for 48 weeks in genotype 1 patients.

The need for new therapies was clear. Over the last decade, significant advancement in our understanding of the HCV life cycle has led to the development of directly acting antivirals
(DAAs), which are focused on three drug-targets that halt the replication of HCV. They are protease inhibitors (PIs) targeting the NS3A/4A protein, which have names ending in "-evir"; polymerase inhibitors targeting the NS5B polymerase and are named with "-buvir"; and inhibitors of the NS5A polymerase, which are named with "-asvir".

In May 2011, the FDA approved the first-in-class NS3/4A serine PIs boceprevir and telaprevir. Both drugs significantly increased SVR rates in HCV mono- and HIV co-infected patients. However, both PIs only had activity for genotype 1 and required administration with PegIFN/RBV. Response guided therapy (RGT) did allow some patients to shorten therapy to 24 weeks instead of 48 weeks. Nevertheless, high pill burden, and side effects of the PI, PegIFN, and RBV remain as treatment barriers.

In December 2013, the FDA approved the first-in-class NS5B polymerase inhibitor sofosbuvir and second generation NS3/4A PI, simeprevir. The approval of these two DAAs opened another era of HCV treatment. Sofosbuvir therapy is not limited to genotype 1 and IFN-free regimens were first approved for HCV genotypes 2 and 3. In response to new treatment options, the American Association for the Study of Liver Disease (AASLD) and Infectious Disease Society of America (IDSA) collaboratively published recommendations for testing, managing, and treating HCV in January 2014,

Department of Internal Medicine, University of Texas Southwestern Medical Center, Dallas, Texas.

(C) The Author(s) 2015; Published by Mary Ann Liebert, Inc. This Open Access article is distributed under the terms of the Creative Commons Attribution Noncommercial License (http://creativecommons.org/licenses/by-nc/4.0/) which permits any noncommercial use, distribution, and reproduction in any medium, provided the original author(s) and the source are credited. 
Table 1. Current Recommended Treatments and Future Regimens FOR TREATMENT-NAÏVE AND -EXPERIENCED HCV PATIENTS

\begin{tabular}{|c|c|c|c|c|c|c|c|}
\hline Genotype & \multicolumn{7}{|c|}{ Preferred and Alternative } \\
\hline $1 \mathbf{a}$ & \multirow{2}{*}{\multicolumn{2}{|c|}{ SOF/LDV 12 wk $^{\mathrm{a}}$}} & \multirow{2}{*}{\multicolumn{3}{|c|}{$\begin{array}{c}\mathrm{PAR} / \mathrm{r}+\mathrm{OMB}+\mathrm{DAS}+\mathrm{RBV} 12 \mathrm{wk}^{\mathrm{b}} \\
\mathrm{PAR} / \mathrm{r}+\mathrm{OMB}+\mathrm{DAS} 12 \mathrm{wk}^{\mathrm{c}}\end{array}$}} & \multirow{2}{*}{\multicolumn{2}{|c|}{$\begin{array}{c}\text { SOF }+ \text { SMV } \pm R B V \\
12 \text { wk or } 24 \text { wk (if cirrhosis) }\end{array}$}} \\
\hline $1 \mathbf{b}$ & & & & & & & \\
\hline \multirow[t]{2}{*}{1} & \multicolumn{7}{|c|}{$\mathrm{SOF}+\mathrm{DCV} \pm \mathrm{RBV} 12-24 \mathrm{wk}^{\mathrm{g}, \mathrm{h}}$} \\
\hline & \multicolumn{7}{|c|}{$\mathrm{GRA}+\mathrm{ELB} \pm \mathrm{RBV}$ for $12 \mathrm{wk}^{\mathrm{h}}$} \\
\hline 2 & \multicolumn{4}{|c|}{ SOF + RBV 12 wk or 16 wk (if cirrhosis) } & \multicolumn{3}{|c|}{$S O F+P e g I F N+R B V 12 w k^{\mathrm{e}}$} \\
\hline \multirow[t]{3}{*}{3} & \multicolumn{4}{|c|}{ SOF+RBV 24 wk } & \multicolumn{3}{|c|}{$S O F+P e g I F N+R B V 12 w k$} \\
\hline & \multicolumn{7}{|c|}{$\mathrm{SOF} / \mathrm{LDV}+\mathrm{RBV} 12 \mathrm{wk}^{\mathrm{h}}$} \\
\hline & \multicolumn{7}{|c|}{$\mathrm{SOF}+\mathrm{DCV} \pm \mathrm{RBV} 12-24 \mathrm{wk}^{\mathrm{g}, \mathrm{h}}$} \\
\hline \multirow[t]{2}{*}{4} & $\begin{array}{l}\text { SOF/LDV } \\
12 \mathrm{wk}^{\mathrm{h}}\end{array}$ & $\mathbf{P A R} /$ & $\begin{array}{l}\text { MB + RBV } \\
\mathbf{w k}^{\mathrm{h}}\end{array}$ & $\begin{array}{c}\text { SOF }+ \text { RBV } \\
24 \text { wk }\end{array}$ & SOF & $\begin{array}{l}P e g I F N+R B V \\
12 w k\end{array}$ & $\begin{array}{c}S O F+S M V \pm R B V \\
12 w k^{\mathrm{f}, \mathrm{h}}\end{array}$ \\
\hline & \multicolumn{7}{|c|}{$\mathrm{SOF}+\mathrm{DCV} \pm \mathrm{RBV} 12-24 \mathrm{wk}^{\mathrm{g}, \mathrm{h}}$} \\
\hline 5 & \multicolumn{4}{|c|}{ SOF +PegIFN+RBV $12 w^{h}$} & \multicolumn{3}{|c|}{$P e g I F N+R B V$ for $48 w k$} \\
\hline 6 & \multicolumn{4}{|c|}{ SOF/LDV $12 w^{\mathrm{h}}$} & \multicolumn{3}{|c|}{$S O F+P e g I F N+R B V 12 w k^{\mathrm{h}}$} \\
\hline
\end{tabular}

Bold indicates preferred by IDSA/AASLD guidance; italics indicates alternative regimens.

DAS, dasabuvir; DCV, daclatasvir; ELB, elbasvir; GRA, grazoprevir; LDV, ledipasvir; OMB, ombitasavir; PAR, paritaprevir; PegIFN, pegylated interferon; R, ritonavir; RBV, ribavirin; SMV, simeprevir; SOF, sofosbuvir.

24 weeks if both cirrhotic and treatment-experienced.

${ }_{2} 4$ weeks for cirrhotic patients regardless of treatment experiences.

${ }^{\mathrm{c}}$ Weight-based RBV is required if cirrhotic regardless of treatment experiences.

${ }^{\mathrm{d}} \mathrm{RBV}$ is optional but in treatment-naïve, GT1b patient, RBV is not recommended.

${ }^{\mathrm{e}}$ Consider this regimen only in treatment-experienced.

${ }^{\mathrm{f}}$ Only in treatment-naïve.

${ }^{\mathrm{g}}$ European guideline.

${ }^{\mathrm{h}}$ Not FDA approved.

with revisions in December $2014 .^{7}$ Major side effects of PegIFN include flu-like symptoms, fatigue, depression, cytopenia, and rash. RBV-related toxicities include anemia, fatigue, irritability, and insomnia. Because of these toxicities, PegIFN is no longer recommended in any treatment regimen for genotype 1 patients. The goal of therapy is to use an alloral regimen when possible and shorter therapy when available (Table 1). A HCV RNA can be obtained at 4 weeks to measure compliance but no stopping rules apply in current recommended regimens. A complete blood count should be taken at weeks 2 and 4, and then monthly to evaluate for anemia if using a regimen containing RBV. As most relapses occur within the first 4 weeks post-treatment, a 4-week posttreatment HCV RNA can be obtained. However, SVR is measured at 12 weeks post-treatment. No detectable virus 12 weeks after completing a course of treatment is equivalent to a cure.

\section{Genotype 1}

\section{Sofosbuvir/ledipasvir}

The fixed dose combination of sofosbuvir/ledipasvir, a combination of an NS5B with an NS5A polymerase inhibitor, was FDA approved in October 2014. The phase 3 ION studies of this fixed-dose combination demonstrate SVR of $94 \%$ for 8 weeks of sofosbuvir/ledipasvir, 93\% for sofosbuvir/ ledipasvir plus RBV for 8 weeks, and $95 \%$ with sofosbuvir/ ledipasvir for 12 weeks in treatment-naïve, non-cirrhotic patients. ${ }^{8}$ In treatment-experienced patients, the SVR was $94 \%$ with 12 weeks of sofosbuvir/ledipasvir, $96 \%$ in those who received sofosbuvir/ledipasvir plus RBV for 12 weeks, and $99 \%$ in those receiving the fixed dose combination with or without RBV for 24 weeks. ${ }^{9}$ Sofosbuvir/ledipasvir has been approved for 8 weeks in treatment-naïve, non-cirrhotic patients, with HCV RNA $<6$ million copies/ml or 12 weeks in treatment-naïve cirrhotic patients or those with $\mathrm{HCV}$ RNA $>6$ million copies/mL. For treatment-experienced cirrhotic patients, the regimen should be extended to 24 weeks. This is the first FDA-approved regimen for HCV genotype 1 that does not require PegIFN/RBV. Ledipasvir solubility decreases as $\mathrm{pH}$ increases, increased gastric $\mathrm{pH}$ is expected to decrease ledipasvir concentration. $\mathrm{H} 2$ blockers should be taken $12 \mathrm{~h}$ apart and proton-pump inhibitors should be avoided. $^{10}$

For HCV/HIV co-infected patients, a study conducted by NIAID/CC Hepatitis C ERADICATE team in this population reports findings of $97-100 \%$ SVR in both ART treated and untreated patients. ${ }^{11} \mathrm{PK}$ data of sofosbuvir/ledipasvir from ION-4 study shows ledipasvir can be co-administered with raltegravir, tenofovir/emtricitabine/efavirenz, or rilpivirine 


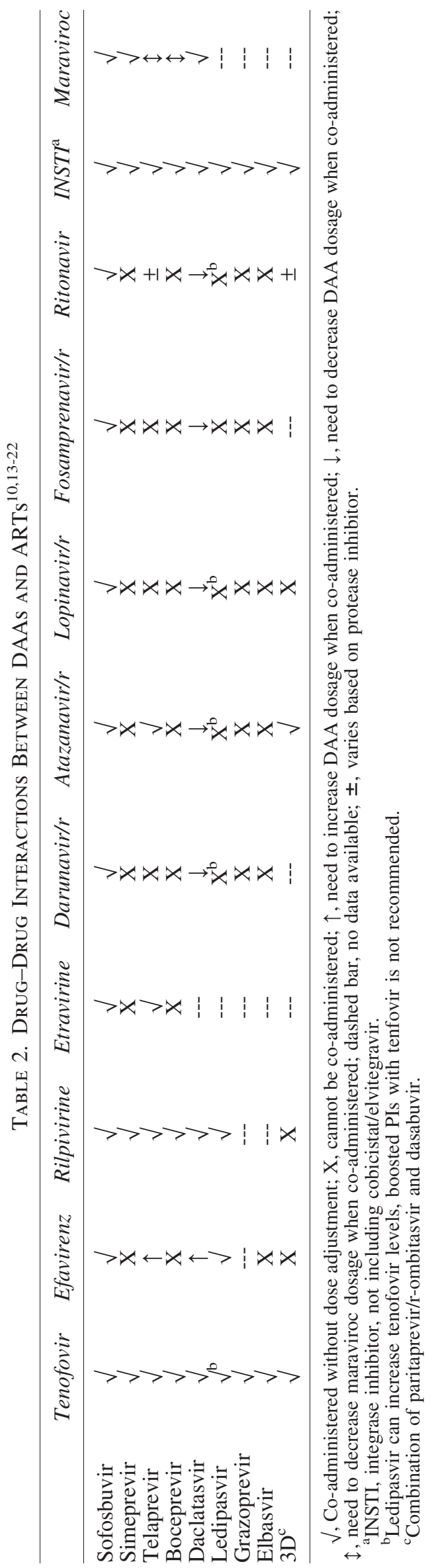

without dose adjustment, but patients taking tenofovir/ emtricitabine/efavirenz should be monitored for tenofovir toxicity. ${ }^{12}$ Based on the package insert, a HIV boosted PI or cobicistat-containing regimen used with tenofovir is not recommended due to lack of safety data on increased tenofovir concentration (see Table 2 for drug-drug interactions). ${ }^{10,13-22}$ Further PK data of sofosbuvir/ledipasvir with boosted PIs and cobicistat/elvitegravir are pending. Ledipasvir is minimally metabolized and primarily eliminated through the gut. $^{23}$ Thus, sofosbuvir/ledipasvir could also be used in patients with mild to moderate renal dysfunction (GFR $>30$ $\mathrm{mL} / \mathrm{min})$.

\section{Sofosbuvir plus simeprevir}

In November 2014, simeprevir was approved by FDA to be used in combination with sofosbuvir. Simeprevir and sofosbuvir should be used for 12 weeks for non-cirrhotic patients and 24 weeks in cirrhotic patients, regardless of previous treatment experiences. In the COSMOS study, sofosbuvir plus simeprevir with or without RBV for 12 weeks yielded SVR12 of $96 \%$ in patients with mild fibrosis (Metavir F0-2) and $86 \%$ in patients with advanced fibrosis (Metavir F3-4). The SVR was 100\% with the same regimen for 24 weeks in patients with cirrhosis; however, only 10 patients were enrolled. ${ }^{24}$

No data from clinical trials exist about the combination of sofosbuvir and simeprevir in HIV-infected patients. Although it would be expected to yield results similar to those seen in mono-infected patients, the drug-drug interactions between simeprevir and many ART regimens would affect administration in co-infected patients. Simeprevir is metabolized through CYP3A and its concomitant use with boosted HIV PIs, efavirenz, etravirine, and tenofovir/emtricitabine/cobicistat/ elvitegravir is not recommended; it may be administered with raltegravir, rilpivirine, maraviroc, and tenofovir. ${ }^{21,22}$ Sofosbuvir is a substrate of drug transporters P-gp, so drugs that are potent P-gp inducers such as rifampin and St. John's Wort are contraindicated. Sofosbuvir has no significant interaction with most ART including tenofovir, emtricitabine, efavirenz, rilpivirine, and darunavir. ${ }^{25}$ Boosted tipranavir, which decreases the concentration of sofosbuvir, is contraindicated. ${ }^{13}$ Caution should be used when administering simeprevir and sofosbuvir in patients with Child Pugh class B and C cirrhosis, as there are no data on drug levels in this population.

\section{Paritaprevir/r-ombitasvir and dasabuvir (3D)}

Another IFN-free all-oral therapy, which obtained FDA approval in December 2014, is a combination of fixed-dose ombitasvir (NS5A inhibitor)/paritaprevir (NS3/4A PI) boosted with ritonavir daily and dasabuvir (NS5B inhibitor) twice daily, also known as 3D, given for $12-24$ weeks. It is recommended to treat genotype 1a without cirrhosis with the $3 \mathrm{D}$ regimen and $\mathrm{RBV}$ for 12 weeks, but extend for 24 weeks in those with cirrhosis. Genotype $1 \mathrm{~b}$ patients without cirrhosis can be treated with the $3 \mathrm{D}$ regimen alone for 12 weeks and those with cirrhosis can be treated with $3 \mathrm{D}$ regimen and RBV for 12 weeks. $^{7}$

In clinical trials when combined with $\mathrm{RBV}$, this regimen produced $\geq 96 \%$ SVR in treatment-naïve and -experienced patients. ${ }^{26,27}$ The most common adverse events were headache, fatigue, and pruritus. Limited PK data show no drug interactions with tenofovir, emtricitabine, atazanavir, or raltegravir. The 
3D regimen is not recommended with efavirenz, lopinavir/ ritonavir, or rilpivirine. Additional studies are being conducted with darunavir. The TURQUOISE-1 trial which enrolled HCV/HIV co-infected patients on either atazanavir or raltegravir regimen treated with $3 \mathrm{D}+\mathrm{RBV}$ for 12 or 24 weeks has SVR12 of $94 \% .^{28}$ Dolutegravir is not expected to have drug interactions.

\section{FDA approved but no longer recommended}

Simeprevir. Simeprevir is a second-generation PI administered once daily at a dose of $150 \mathrm{mg}$. The data indicate that, in patients who did not meet RGT criteria, extension of treatment to 48 weeks did not significantly improve SVR. ${ }^{29,30}$ Thus, the drug is administered with PegIFN/RBV for 12 weeks, followed by PegIFN/RBV alone to complete 24 weeks of treatment in genotype $1 \mathrm{~b}$ or genotype 1a without Q80K polymorphism. ${ }^{14}$ Pooled overall response rates from QUEST 1 and QUEST 2 showed an $80 \%$ SVR compared with $50 \%$ in the placebo group. ${ }^{31}$

In HIV-infected patients, Study C212 enrolled treatmentnaïve and -experienced patients and achieved overall SVR of $74-79 \%$ in treatment-naïve, $87 \%$ in prior relapsers, $70 \%$ in partial responders, and $57 \%$ in null responders. Overall SVR rates were $80 \%$ for patients with mild fibrosis and $64 \%$ for those with advanced fibrosis. ${ }^{32}$ Approximately $28 \%$ of patients had the Q80K polymorphism that has been shown to reduce SVR in HCV mono-infected patients treated with simeprevir, ${ }^{14}$ but this was not found in co-infected patients.

Sofosbuvir. Sofosbuvir is a NS5B polymerase inhibitor formulated as a $400 \mathrm{mg}$ tablet taken once daily. In treatmentnaïve patients, sofosbuvir combined with PegIFN and RBV is given for 12 weeks. Sofosbuvir has been studied mostly in treatment-naïve patients with genotype 1 . In the phase 3 NEUTRINO trial, 90\% SVR was achieved in treatment-naïve patients with sofosbuvir plus PegIFN/RBV for 12 weeks; but less than $20 \%$ of the patients had cirrhosis. ${ }^{33}$ Unlike PegIFN/ $\mathrm{RBV}$, in which cirrhotic patients had significantly lower SVR, cirrhotic patients in the NEUTRINO study had an $80 \%$ SVR compared with a $92 \%$ SVR in non-cirrhotic patients treated with sofosbuvir, PegIFN/RBV for genotype 1 and $4 .{ }^{33}$

The phase 2 ELECTRON trial was the only study to examine an IFN-free regimen of sofosbuvir and RBV for 12 weeks in treatment-experienced patients (prior nonresponders). SVR in this difficult-to-treat population was only $10 \%$. More data on treatment-experienced patients were not available. Therefore, the FDA performed a post-hoc analysis and estimated that the response rate for genotype 1 prior non-responders would be approximately $71 \%$ with sofosbuvir/PegIFN/RBV based on the NEUTRINO study, using treatment-naïve patients with unfavorable characteristics such as advanced fibrosis, IL28B non-CC subgenotype, and high HCV RNA viremia. ${ }^{13}$

In $\mathrm{HCV} / \mathrm{HIV}$ co-infected patients, the response rate to sofosbuvir is very similar to HCV mono-infected patients. Sofosbuvir plus PegIFN/RBV for 12 weeks resulted in SVR of $89 \% .{ }^{34}$ A 24 -week course of sofosbuvir and RBV yielded an SVR of $75 \%$ (82\% for genotype 1a and 54\% for genotype 1b) for co-infected patients in the PHOTON study, and should not be considered due to the lower SVR. ${ }^{35}$ In the PHOTON study, among 31 patients taking boosted atazanavir,
$77 \%$ developed grade 3 hyperbilirubinemia, and 5 patients required a change in ART. Indirect hyperbilirubinemia, a common laboratory abnormality in HIV patients who are on atazanavir, results from an inhibitory competition by atazanavir of the uridinglucuronosyltransferase (UGT) 1A1 enzyme, which is responsible for bilirubin conjugation. Indirect hyperbilirubinemia is increased in patients who are also incurring hemolysis resulting from RBV. ${ }^{36}$ Hyperbilirubinemia will resolve once treatment is completed and there is no evidence of direct liver damage resulting from coadministration of this $\mathrm{HCV}$ regimen with boosted atazanavir.

Boceprevir. Treatment with boceprevir requires a 4-week PegIFN/RBV induction phase followed by a 24- or 44-week course of PegIFN/RBV and boceprevir, depending on virological response. ${ }^{16}$ The PO5411 Trial in HCV/HIV coinfected treatment-naïve patient obtained an SVR of $63 \%$ (40/64) compared with $29 \%$ (10/34) among those who received PegIFN/RBV alone. ${ }^{37}$ This SVR rate was similar to what was seen among HCV mono-infected patients. This was the first data demonstrating that combination treatment with DAAs in the co-infected population resulted in SVR rates similar to those in the mono-infected population. Subsequent studies including $\mathrm{C} 110$, PHOTON, and C212 with new DAAs appear to confirm this initial finding. ${ }^{32,35,38}$

Because boceprevir is a strong inhibitor of CYP3A and partially metabolized by this pathway, there are several drugdrug interactions. It is not recommended to use boceprevir with ritonavir-boosted PIs such as darunavir, atazanavir, or lopinavir $^{39-41}$ or with efavirenz or etravirine. ${ }^{39,42}$ Drugs that may be used with boceprevir include rilpivirine, dolutegravir, raltegravir, maraviroc, and tenofovir. ${ }^{39,43-47}$ Because of the numerous drug-drug interactions, longer course of therapy, and side effects of the combination of medications, boceprevir should no longer be used.

Telaprevir. Similar to boceprevir, the IDSA/AASLD guidelines do not recommend the use of telaprevir in $\mathrm{HCV} /$ HIV co-infected patients. ${ }^{7}$ Telaprevir is administered in combination with PegIFN/RBV for 12 weeks, followed by a RGT of an additional 12 or 36 weeks of PegIFN/RBV in treatment naïve. RGT is not recommended in cirrhotic or treatmentexperienced patients, therefore a 48-week course of therapy is needed in this population. ${ }^{15}$

In HCV/HIV co-infected patients, the phase 2a Study C110 enrolled 38 treatment-naïve patients who received a total of 48 weeks of treatment and achieved an SVR of $74 \% .{ }^{38}$ The phase 3 INSIGHT study included 162 co-infected patients showed SVR ranging from $84 \%$ in treatment-naïve patients to $41 \%$ in prior null-responders. ${ }^{48}$

Telaprevir is also a strong inhibitor of CYP3A4 and a substrate of P-gp. It cannot be administered with darunavir, lopinavir, or fosamprenavir, and when it is co-administered with efavirenz, the dose of telaprevir should be increased from $750 \mathrm{mg}$ TID to $1125 \mathrm{mg}$ TID. ${ }^{49-51}$ Telaprevir can be safely given with etravirine, rilpivirine, dolutegravir, raltegravir, and maraviroc (with a dosage decrease to $150 \mathrm{mg}$ BID). ${ }^{44,46,47,52}$

Telaprevir has a black box warning of serious skin reaction including fatal and non-fatal Stevens Johnson Syndrome, toxic epidermal necrolysis, and drug reaction with eosinophilia and 
systemic symptoms (DRESS), which occurs in less than $1 \%$ of patients. Rash (all severity) developed in $56 \%$ of patients who received telaprevir. ${ }^{15}$

\section{Drugs not yet FDA approved}

Daclatasvir. Daclatasvir is a nonstructural protein NS5A inhibitor that has pan-genotypic activity and was approved in Europe in August 2014. It is expected to be available in the US by the end of 2015. Daclatasvir has been studied in combination with PegIFN/RBV in the COMMAND-1 study, which yielded an SVR of $65 \%$ in genotype 1 patients. ${ }^{53}$ In the European Guidelines, daclatasvir plus PegIFN/RBV for 24 weeks is recommended especially for genotype $1 b .{ }^{54}$ The dose of daclatasvir needs to be decreased to $30 \mathrm{mg}$ when administered with boosted PIs and increased to $90 \mathrm{mg}$ when administered with efavirenz. ${ }^{55}$ Daclatasvir does not need to be adjusted for any renal impairment.

Daclatasvir and sofosbuvir. When approved in the US, daclatasvir is likely to be used in combination with another DAA, such as sofosbuvir. In HCV mono-infection, daclatasvir $60 \mathrm{mg}$ daily and sofosbuvir $400 \mathrm{mg}$ daily with or without RBV achieved 98\% SVR in treatment-naïve for 12 or 24 weeks. Treatment-experienced patients who had failed previous HCV PI therapy were treated for 24 weeks and achieved a similar response. ${ }^{56}$ In HIV co-infected patient, ALLY-2 demonstrated greater than 96\% SVR when daclatasvir and sofosbuvir were administered for 12 weeks in genotype 1-4 regardless of treatment experience. Cirrhotic patients have lower SVR, around $91 \%{ }^{57}$ In Europe, daclatasvir and sofosbuvir is recommended for 12 weeks for noncirrhotic patients and 24 weeks for compensated cirrhotic patients. ${ }^{54}$

Grazoprevir (MK-5172) and elbasvir (MK-874). Grazoprevir is another once daily NS3/4A PI. Following RGT, grazoprevir plus PegIFN/RBV achieved SVR rates $>90 \%$ in HCV mono-infected patients. ${ }^{58}$ An IFN-free combination grazoprevir with elbasvir (an NS5A inhibitor) with or without RBV is now in phase 3 development. In the phase 2 C-WORTHY study, grazoprevir and elbasvir with or without RBV for 12 weeks, led to SVR of 95\% in HCV mono-infected patients. In part B of the C-WORTHY trial, 59 HCV/HIV co-infected patients on an NRTI plus raltegravir were evaluated for grazoprevir/elbasvir with or without RBV for 12 weeks. SVR was $96 \%$ for patients receiving this regimen with RBV and $89 \%$ in patients receiving the regimen without RBV. ${ }^{59}$

A recent PK study showed that grazoprevir cannot be used with boosted PIs, ${ }^{60}$ and that there is no interaction between grazoprevir and either tenofovir or raltegravir. ${ }^{20}$ Elbasvir does not interact with tenofovir or raltegravir but co-administration does lead to decreased levels of elbasvir when combined with efavirenz. ${ }^{19}$ Ritonavir-boosted lopinavir, atazanavir, and darunavir increased levels of elbasvir, therefore should not be co-administered. ${ }^{18}$ Dolutegravir can be used with these drugs, but additional data is needed on rilpivirine.

Daclatasvir and asunaprevir. The combination of daclatasvir (NS5A inhibitor) with asunaprevir (NS3/4A PI) showed an SVR of $84 \%$ in cirrhotic patients and $85 \%$ in noncirrhotic genotype $1 \mathrm{~b}$ patients. Among cirrhotic patients, SVR was $91 \%$ in treatment naïve, $87 \%$ in prior non-responders and $81 \%$ in IFN-ineligible/intolerant groups. ${ }^{61}$ This regimen was approved in Japan in July 2014 because of the high prevalence of $1 \mathrm{~b}$. Asunaprevir is not being pursued for FDA approval in the US.

Faldaprevir. Faldaprevir, a second-generation PI, has been recently withdrawn from further drug development due to the rapidly changing $\mathrm{HCV}$ market in which all oral therapy is an expectation. STARTVERSO 4 used faldaprevir with PegIFN/RBV with RGT and was the largest HCV/HIV coinfected treatment trial. Faldeprevir $240 \mathrm{mg}$ was used with efavirenz-based ART and $120 \mathrm{mg}$ with boosted-PIs, raltegravir, and marviroc. The overall SVR was $72 \% .^{62}$

\section{Genotype 2}

The IDSA/AASLD recommendations support the use of sofosbuvir and RBV for 12 weeks in genotype 2 patients who are HCV treatment-naïve or-experienced and extended to 16 weeks if the patient has cirrhosis. Sofosbuvir plus RBV for 12 weeks achieved SVR rates greater than 95\% in treatmentnaïve patients in both the FISSION and VALENCE studies. ${ }^{33,63}$ SVR for treatment-experienced genotype 2 patients were $90 \%$ in the VALENCE study and $82 \%$ in the FUSION study. ${ }^{63,64}$ In cirrhotic patients, SVR for genotype 2 patients treated with sofosbuvir and RBV for 12 weeks were $100 \%$ and $88 \%$ for treatment-naïve and -experienced patients, respectively. In HCV/HIV co-infected patients, sofosbuvir and RBV for 12 weeks achieved SVR of $88 \%$ in treatmentnaïve patients in the PHOTON study. In treatment-experienced patients who received 24 weeks of sofosbuvir and RBV, SVR were $92 \% .{ }^{65}$ Thus, IFN-free treatment for genotype 2 is now considered the standard of care.

\section{Genotype 3}

In genotype 3, sofosbuvir and RBV is recommended for 24 weeks. Sofosbuvir and RBV for 24 weeks led to SVR of $93 \%$ in treatment-naïve patients and $78 \%$ in treatment-experienced patients based on data from the VALENCE study. In genotype 3 cirrhotic patients, sofosbuvir and RBV for 24 weeks had SVR of $92 \%$ for treatment-naïve and $60 \%$ for treatment-experienced. ${ }^{63}$ Alternatively, sofosbuvir plus PegIFN/RBV for 12 weeks may be considered in IFN-eligible patients, which resulted in $83 \%$ SVR in treatmentexperienced mono-infected patients, of whom 55\% were cirrhotic. ${ }^{66}$ Another option now available with limited data is sofosbuvir/ledipasvir with RBV for 12 weeks with SVR of $100 \%$ in treatment-naïve patients and $82 \%$ in treatmentexperienced patients. ${ }^{67,68}$ Additionally, the European Guidelines have added daclatasvir and sofosbuvir for 12 weeks in treatment-naïve and 24 weeks in treatment -experienced patients. In the phase $2 \mathrm{~b}$ trail, daclatasvir and sofosbuvir with or without RBV for 24 weeks in treatment-naïve, non-cirrhotic patients had SVR rate of $89 \% .^{56}$ The ALLY-3 trial using the same regimen without RBV for 12 weeks yield SVR of $90 \%$ in treatment-naïve and $86 \%$ in treatment-experienced patients. ${ }^{69}$

In HCV/HIV co-infected patients, sofosbuvir and RBV for 12 weeks achieved SVR of $67 \%$ in treatment-naïve patients 
in the PHOTON study. In treatment-experienced patients who received 24 weeks of sofosbuvir and RBV, SVR was $94 \% .{ }^{65}$ For genotype 3 patients, a longer course of sofosbuvir and RBV therapy is needed which also incurs a higher cost.

\section{Genotype 4}

Treatment options for genotype 4 have significantly increased based on the revised guidelines. ${ }^{7}$ The PEARL-I study showed SVR of $100 \%$ in both treatment-naive and -experienced patients with ombitasvir and paritaprevir/ritonavir with RBV and SVR of $91 \%$ in treatment-naïve patients with ombitasvir and paritaprevir/ritonavir only. ${ }^{70}$ Clinical trials using sofosbuvir/ledipasvir for 12 weeks for genotype 4 showed SVR of $95 \%$ in 21 patients. ${ }^{71}$ Sofosbuvir plus RBV for 24 weeks has been shown to be highly effective with $100 \%$ SVR in a small Egyptian study. ${ }^{72}$ Alternatively, sofosbuvir plus PegIFN/RBV for 12 weeks had an SVR rate of $96 \%$ in the NEUTRINO study. An alternative regimen for treatmentnaïve is sofosbuvir and simeprevir with or without RBV for 12 weeks, based on in vitro and in vivo activity of simeprevir for genotype 4; clinical studies are planned. ${ }^{54}$ Also daclatasvir and sofosbuvir for 12 weeks in treatment-naïve and 24 weeks in treatment-experienced can be considered.

Currently, there are no data on DAAs in HCV/HIV coinfected genotype 4 patients. However, the above treatment regimens for $\mathrm{HCV}$ mono-infected can be applied to coinfected patients with the caution regarding DAA and HAART interactions.

\section{Genotypes 5 and 6}

IDSA/AASLD guidelines recommend PegIFN, RBV, and sofosbuvir for 12 weeks for interferon-eligible patients in genotype 5. The NEUTRINO study showed $100 \%$ SVR in genotype $5(n=1)$ and $6(n=6)$ patients. ${ }^{33}$ For genotype 6, sofosbuvir/ledipasvir for 12 weeks is recommended based on limited data showing SVR of $96 \% .^{68}$ European guidelines recommend use of sofosbuvir and RBV for 24 weeks in interferon-ineligible patients.

\section{Real-World Experience}

Based on the experience with teleparevir and boceprevir, it is now known that the real world SVR data are slightly lower than clinical trials. ${ }^{73}$ It has also shown that the uptake of triple therapy treatment in HCV/HIV patients is low (39\%), even in treatment-eligible patients. ${ }^{74}$ This points out the difficulties in treating HCV/HIV patients in the real world. Since the approval of sofosbuvir and simeprevir in late 2013, data on using these DAA in the real world have been presented, including in HIV patients. Data from the TRIO network show that simeprevir/sofosbuvir with or without RBV for 12 weeks in genotype $1 \mathrm{HCV}$ mono-infected patients had SVR of $82 \%$ and sofosbuvir plus RBV for 12 weeks for genotype 2 patients had SVR of $84 \% .{ }^{75}$ Similar data from HCV-TARGET showed SVR at post-treatment week 4 of $89 \%$ in simeprevir/ sofosbuvir with or without RBV for 12 weeks in genotype 1 patients. ${ }^{76}$ In both real-world data, there are no significant differences between regimens with or without RBV. One single center treated $22 \mathrm{HIV} / \mathrm{HCV}$ co-infected patients with sofosbuvir/simeprevir with or without RBV reported SVR post-treatment week 4 of $96 \% .{ }^{77}$ In the real world, it appears that SVR is approximately $10 \%$ lower than SVR reported from clinical trials, but long-term data are needed.

The advancement of HCV treatment is reminiscent of the evolution of HIV therapy, albeit at a much faster pace. Moving forward, an NS5A or NS5B polymerase inhibitor will need to be the backbone of the regimen (similar to NRTI in HIV regimens). In HCV/HIV co-infected patients, ART should be initiated or adjusted based on preferred HCV treatment and the potential for drug-drug interactions. $\mathrm{Nu}-$ merous treatment options will likely soon be available for the $\mathrm{HCV} / \mathrm{HIV}$ co-infected patient, but important drug-drug interactions studies are still needed so that patients may be safely treated without risking HIV virologic failure or $\mathrm{HCV}$ drug toxicity. In time, these drugs will help to increase life expectancy in those patients living with both HIV and HCV.

\section{Acknowledgments}

The article was originally supported by Boehringer Ingelheim and subsequently independently developed by the authors.

\section{Author Disclosure Statement}

Dr. Jain reports grants from Bristol-Myers Squibb, grants, personal fees, and nonfinancial support from Gilead Sciences, grants from Janssen Pharmaceuticals, grants, personal fees, and nonfinancial support from Boehringer Ingelheim, and grants and personal fees from AbbVie, outside the submitted work.

Dr. Chen has no conflicting financial interests to disclose.

\section{References}

1. Ly KN, Xing J, Klevens RM, Jiles RB, Ward JW, Holmberg SD. The increasing burden of mortality from viral hepatitis in the United States between 1999 and 2007. Ann Intern Med 2012;156:271-278.

2. Lohse N, Hansen AB, Pedersen G, et al. Survival of persons with and without HIV infection in Denmark, 1995-2005. Ann Intern Med 2007;146:87-95.

3. Schwarcz SK, Vu A, Hsu LC, Hessol NA. Changes in causes of death among persons with AIDS: San Francisco, California, 1996-2011. AIDS Patient Care STD 2014;28: 517-523.

4. Torriani FJ, Rodriguez-Torres M, Rockstroh JK, et al. Peginterferon Alfa-2a plus ribavirin for chronic hepatitis $\mathrm{C}$ virus infection in $\mathrm{HIV}$-infected patients. $\mathrm{N}$ Engl $\mathrm{J}$ Med 2004;351:438-450.

5. Moreno A, Quereda C, Moreno L, et al. High rate of didanosine-related mitochondrial toxicity in HIV/HCVco-infected patients receiving ribavirin. Antivir Ther 2004;9: $133-138$

6. Alvarez D, Dieterich DT, Brau N, Moorehead L, Ball L, Sulkowski MS. Zidovudine use but not weight-based ribavirin dosing impacts anaemia during HCV treatment in HIV-infected persons. J Viral Hepat 2006;13:683-689.

7. IDSA/AASLD: Recommnedations for testing, managing, and treating hepatitis C. http://www.hcvguidelines.org 2014 (Last accessed April 2, 2015).

8. Kowdley KV, Gordon SC, Reddy KR, et al. Ledipasvir and sofosbuvir for 8 or 12 weeks for chronic $\mathrm{HCV}$ without cirrhosis. N Engl J Med 2014;370:1879-1888. 
9. Afdhal N, Reddy KR, Nelson DR, et al. Ledipasvir and sofosbuvir for previously treated HCV genotype 1 infection. N Engl J Med 2014;370:1483-1493.

10. FDA approved drug products: Ledipasvir and Sofosbuvir.

11. Townsend KOA, Nelson A, et al. High efficacy of sofosbuvir/ledipasvir for the treatment of HCV genotype 1 in patients co-infected with HIV on or off antiretroviral therapy: Results from the NIAID ERADICATE trial. The American Association for the Study of Liver Diseases, November 9-13, 2014, Boston, MA.

12. German P, Pang P, West S, et al. Drug interactions between direct-acting anti-HCV antivirals sofosbuvir and ledipasvir and HIV antiretrovirals. 15th International Workshop on Clinical Pharmacology of HIV and Hepatitis Therapy, May 19-21, 2014, Washington, DC.

13. FDA approved drug products: Sofosbuvir. http://www .accessdata.fda.gov/drugsatfda_docs/label/2013/204671s000lbl .pdf (Last accessed April 2, 2015).

14. FDA approved drug products: Simeprevir. http://www .accessdata.fda.gov/drugsatfda_docs/label/2013/205123s001lbl .pdf (Last accessed April 2, 2015).

15. FDA approved drug products: Telaprevir. http://www .accessdata.fda.gov/drugsatfda_docs/label/2013/201917s012lbl .pdf (Last accessed April 2, 2015).

16. FDA approved drug products: Boceprevir. http://www .accessdata.fda.gov/drugsatfda_docs/label/2014/202258s015lbl .pdf (Last accessed April 2, 2015).

17. Bifano M, Hwang C, Oosterhuis B, et al. Assessment of pharmacokinetic interactions of the HCV NS5A replication complex inhibitor daclatasvir with antiretroviral agents: Ritonavir-boosted atazanavir, efavirenz and tenofovir. Antivir Ther 2013;18:931-940.

18. Yeh W, Marshal W, Ma J, et al. Ritonavir-boosted atazanavir, lopinavir and darunavir increase HCV NS5A inhibitor MK-8742 levels. 21st Conference On Retroviruses And Opportunistic Infections, March 3-6, 2014, Boston MA.

19. Yeh W, Marshal W, Mangin E, et al. Pharmacokinetic interactions between the HCV NS5A inhibitor MK-8742 and efavirenz. 21st Conference on Retroviruses and Opportunistic Infections, 2014, Boston MA.

20. Yeh W, Fraser I, Caro L, et al. No meaningful PK interaction between HCV protease inhibtor MK-5172 and tenofovir or raltegravir. 21st Conference on Retroviruses and Opportunistic Infections, March 3-6, 2014, Boston MA.

21. Ouwerkerk-Mahadevan S, Sekar V, Simion A, et al. The pharmacokinetic interactions of the $\mathrm{HCV}$ protease inhibitor simeprevir (TMC435) with HIV antiretroviral agents in healthy volunteers. 2012 Infectious Diseases Society of America (IDSA), October 17-21, 2012, San Diego.

22. Ouwerkerk-Mahadevan S, Simion A, Peeters M, et al. Summary of pharmacokinetic drug-drug interactions for simeprevir (TMC435), a hepatitis C virus NS3/4A protease inhibitor. 14th European AIDS Conference, October 16-19, 2013, Brussels, Belgium.

23. Kirby B, Mathias A, Yang C. Metabolism and excretion of ledipasvir. 8th International Workshop on Clinical Pharmacology of Hepatitis Therapy, June 26-27, 2013, Boston MA.

24. Lawitz E, Sulkowski MS, Ghalib R, et al. Simeprevir plus sofosbuvir, with or without ribavirin, to treat chronic infection with hepatitis $\mathrm{C}$ virus genotype 1 in non-responders to pegylated interferon and ribavirin and treatment-naive patients: The COSMOS randomised study. Lancet 2014; 384:1756-1765.
25. Kirby B, Mathias A, Rossi S, Moyer C, Shen G, Kearney BP. No clinically significant pharmacokinetic drug interactions between sofosbuvir and HIV antiretroviral Atripla, rilpivirine, darunaivr/ritonavir, or rlategravir in healthy volunteers. 64th Annual Meeting of the American Association for the Study of Liver Diseases, November 1-5, 2013, Washington DC.

26. Zeuzem S, Jacobson IM, Baykal T, et al. Retreatment of HCV with ABT-450/r-ombitasvir and dasabuvir with ribavirin. N Engl J Med 2014;370:1604-1614.

27. Feld JJ, Kowdley KV, Coakley E, et al. Treatment of HCV with ABT-450/r-ombitasvir and dasabuvir with ribavirin. N Engl J Med 2014;370:1594-1603.

28. Wyles D, Sulkowski M, Eron J, et al. TURQUOISE-I: 94\% SVR12 in HCV/HIV-1 co-infected patients treated with ABT-450/r/Ombitasvir, Dasabuvir and ribavirin. American Association for the Study of Liver Diseases, 2014, Boston, MA.

29. Manns M, Marcellin P, Poordad F, et al. Simeprevir with pegylated interferon alfa $2 \mathrm{a}$ or $2 \mathrm{~b}$ plus ribavirin in treatment-naive patients with chronic hepatitis $\mathrm{C}$ virus genotype 1 infection (QUEST-2): A randomised, double-blind, placebo-controlled phase 3 trial. Lancet 2014;384:414426.

30. Jacobson IM, Dore GJ, Foster GR, et al. Simeprevir with pegylated interferon alfa 2 a plus ribavirin in treatmentnaive patients with chronic hepatitis $C$ virus genotype 1 infection (QUEST-1): A phase 3, randomised, double-blind, placebo-controlled trial. Lancet 2014;384:403-413.

31. Jacobson IM, Dore GJ, Foster GR, et al. Simeprevir (TMC435) with peginterferon/ribavirin for treatment of chronic HCV genotype 1 infection in treatment-naïve patients: Efficacy in difficult-to-treat patient sub-populations in the QUEST-1 and 2 Phase III trials. 64th Annual Meeting of the American Association for the Study of Liver Diseases (AASLD), November 1-5, 2013, Washington, DC.

32. Dieterich D, Rockstroh J, Orkin C, et al. Simeprevir (TMC435) plus PegIFN/Ribavirin in HCV genotype-1/ HIV-1 co-infection (Study C212). March 3-6, 2014, Boston MA.

33. Lawitz E, Mangia A, Wyles D, et al. Sofosbuvir for previously untreated chronic hepatitis $\mathrm{C}$ infection. $\mathrm{N}$ Engl $\mathrm{J}$ Med 2013;368:1878-1887.

34. Rodriquez-Torres M, Rodriguez-Orengo J, Gaggar A, et al. Sofosbuvir and peginterferonn alfa-2a/ribavirin for treatment-naive genotype 1-4 $\mathrm{HCV}$-infected patients who are co-infected with HIV. ID Week, October 2-6, 2013, 2013, San Francisco, CA.

35. Sulkowski MS, Naggie S, Lalezari J, et al. Sofosbuvir and ribavirin for hepatitis $\mathrm{C}$ in patients with HIV co-infection. JAMA 2014;312:353-361.

36. Rodriguez-Novoa S, Morello J, Gonzalez M, et al. Increase in serum bilirubin in HIV/hepatitis-C virus-co-infected patients on atazanavir therapy following initiation of pegylated-interferon and ribavirin. AIDS 2008;22:25352537.

37. Sulkowski M, Pol S, Mallolas J, et al. Boceprevir versus placebo with pegylated interferon alfa- $2 b$ and ribavirin for treatment of hepatitis $C$ virus genotype 1 in patients with HIV: A randomised, double-blind, controlled phase 2 trial. Lancet Infect Dis 2013;13:597-605.

38. Sulkowski MS, Sherman KE, Dieterich DT, et al. Combination therapy with telaprevir for chronic hepatitis $\mathrm{C}$ virus 
genotype 1 infection in patients with HIV: A randomized trial. Ann Intern Med 2013;159:86-96.

39. Kasserra C, Hughes E, Treitel M. Combination therapy with telaprevir for chronic hepatitis $\mathrm{C}$ virus genotype 1 infection in patients with HIV. Paper presented at 18th Conference on Retroviruses and Opportunistic Infections February 27-March 2, 2011, Boston MA.

40. Hulskotte EG, Feng HP, Xuan F, et al. Pharmacokinetic interactions between the hepatitis $\mathrm{C}$ virus protease inhibitor boceprevir and ritonavir-boosted HIV-1 protease inhibitors atazanavir, darunavir, and lopinavir. Clin Infect Dis 2013; 56:718-726.

41. Garraffo R, Lavrut T, Chen S, et al. Combination therapy with telaprevir for chronic hepatitis $\mathrm{C}$ virus genotype 1 infection in patients with HIV. Combination therapy with telaprevir for chronic hepatitis $\mathrm{C}$ virus genotype 1 infection in patients with HIV, April 22-24, 2013, Amsterdam, Netherland.

42. Hammond KP, Wolfe P, Burton JR, Jr., et al. Pharmacokinetic interaction between boceprevir and etravirine in HIV/HCV seronegative volunteers. J Acquir Immune Defic Syndr 2013;62:67-73.

43. de Kanter CT, Blonk MI, Colbers AP, Schouwenberg BJ, Burger DM. Lack of a clinically significant drug-drug interaction in healthy volunteers between the hepatitis $\mathrm{C}$ virus protease inhibitor boceprevir and the HIV integrase inhibitor raltegravir. Clin Infect Dis 2013;56:300-306.

44. Vourvahis M, Plotka A, Kantaridis C, et al. The effect of boceprevir and telaprevir on the pharmacokinetics of maraviroc: An open-label, fixed-sequence study in healthy volunteers. 14th International HIV Clinical Pharmacology Workshop, April 22-24, 2013, Amsterdam, Netherland.

45. Rhee RG, Feng H, Xuan F, et al. Absence of a significant pharmacokinetic interaction between the $\mathrm{HCV}$ protease inhibitor boceprevir and HIV-1 NNRTI rilpivirine. 20th Conference on Retroviruses and Opportunistic Infections. March 3-6, 2013, Atlanta GA.

46. Moreno A, Quereda C, Montes M, et al. Safe coadministration of raltegravir-based HAART in HIV-infected patients with $\mathrm{HCV}$-cirrhosis receiving triple therapy with telaprevir or boceprevir. J Acquir Immune Defic Syndr 2012;61: e47-e49.

47. Johnson M, Borland J, Chen S, et al. The effect of boceprevir and telaprevir on dolutegravir pharmacokinetics, in healthy adult subjects. 14th International HIV Clinical Pharmacology Workshop, April 22-24, 2013, Amsterdam, Netherland.

48. Montes M, Nelson M, Girard P, et al. Telaprevir combination therapy in treatment-naïve and experienced patients co-infected with HCV and HIV (INSIGHT STUDY): Sustained virologic response at 12 weeks final analysis. The American Association for the Study of Liver Diseases, November 1-5, 2013, Boston, MA.

49. van Heeswijk RP, Vandevoorde A, Boogaerts G. Pharmacokinetic interactions between ARV agents and the investigational HCV protease inhibitor TVR in healthy volunteers. 18th Conference on Retroviruses and Opportunistic Infections, February 27-March 2, 2011, Boston, MA.

50. Henshaw J, Adiwijaya B, Adda N, et al. The pharmacokinetics of telaprevir and selected ART medications in $\mathrm{HCV} /$ HIV co-infected patients. ID Week, October 17-21, 2012, San Diego.

51. Bertelsen K, Montes ML, Horban A. Efficacy, safety and pharmacokinetics of telaprevir, peg-IFN-alfa-2a, and riba- virin in combination with darunavir/ritonavir-based HAART in HCV/HIV-1 co-infected patients (INSIGHT substudy). 14th European AIDS Conference (EACS), October 16-19, 2013, Brussels, Belgium.

52. Kakuda T, Leopold L, Nijs S. Pharmacokinetic interaction between etravirine or rilpivirine and telaprevir in healthy volunteers: A randomised, two-way crossover trial. The 13th International Workshop on Clinical Pharmacology of HIV Therapy, April 16-18, 2012, Barcelona, Spain.

53. Hézode C, Hirschfield GM, Ghesquiere W, et al. Daclatasvir, an NS5A replication complex inhibitor, combined with peginterferon alfa- $2 \mathrm{a}$ and ribavirin in treatment-naive HCV-genotype 1 or 4 subjects: Phase 2b COMMAND-1 SVR12 results. 6th Annual Meeting of the American Association for the Study of Liver Diseases, November 9-13, 2012, Boston MA.

54. European Association for the Study of the Liver. EASL recommendations on treatment of hepatitis C 2014.

55. Bifano M, Hwang C, Oosterhuis B, et al. Assessment of HIV ARV drug interactions with the HCV NS5A replication complex inhibitor BMS-790052 demonstrates a pharmacokinetic profile which supports co-administration with tenofovir disoproxil fumarate, efavirenz, and atazanavir/ ritonavir. 19th Conference on Retroviruses and Opportunistic Infections, March 5-8, 2012, Seattle, WA.

56. Sulkowski MS, Gardiner DF, Rodriguez-Torres M, et al. Daclatasvir plus sofosbuvir for previously treated or untreated chronic HCV infection. N Engl J Med 2014;370:211-221.

57. Wyles DL, Ruane P, Sulkowski M, et al. Daclatasvir in combination with sofosbuvir for $\mathrm{HIV} / \mathrm{HCV}$ coinfection: ALLY-2 study. 22nd Conference on Retroviruses and Opportunistic Infections. February 23-26, 2015, Seattle, WA.

58. Manns MP, Vierling JM, Bacon BR, et al. The combination of MK-5172, peginterferon, and ribavirin is effective in treatment-naive patients with hepatitis $C$ virus genotype 1 infection without cirrhosis. Gastroenterology 2014;147:366-376.

59. Sulkowski M, Hezode C, Gerstoft J, et al. Efficacy and safety of MK-5172+MK8742 with or without RBV in $\mathrm{HCV}$ mono-infected and HIV/HCV co-infected treatmentnaive, non-cirrhotic patients with hepatitis $\mathrm{C}$ virus genotype 1 infection: The C-WORTHY study (Final results, Parts A and B). American Association for the Study of Liver Diseases, November 8-13, 2014, Boston, MA.

60. Caro L, Talaty JE, Guo Z, et al. Pharmacokinetic interactions between the HCV protease inhibitor MK-5172 and ritonavir-boosted HIV protease inhibitors (atazanavir, lopinavir, darunavir) in healthy volunteers. American Association for the Study of Liver Diseases 64th Annual Meeting, November 1-5, 2013, Washington, DC.

61. Kao J-H, Heo J, Yoffe B, et al. Efficacy and safety of daclatasvir in combination with asunaprevir in cirrhotic and non-cirrhotic patients with HCV genotype $1 \mathrm{~b}$ : Results of the HALLMARK-DUAL study. 49th Annual Meeting of the European Association for the Study of the Liver, April 9-13, 2014, London, UK.

62. Dieterich D, Tural C, Nelson M, et al. Faldaprevir plus pegylated interferon $\alpha$-2a/ribavirin in HIV/HCV co-infection: STARTVerso4. CROI 2014, Boston.

63. Zeuzem S, Dusheiko GM, Salupere R, et al. Sofosbuvir and ribavirin in HCV genotypes 2 and 3. N Engl J Med 2014; 370:1993-2001.

64. Jacobson IM, Gordon SC, Kowdley KV, et al. Sofosbuvir for hepatitis $\mathrm{C}$ genotype 2 or 3 in patients without treatment options. N Engl J Med 2013;368:1867-1877. 
65. Naggie S, Sulkowski M, Lalezari J, et al. All-oral therapy with sofosbuvir plus ribavirin for the treatment of HCV genotype 1, 2, and 3 infection in patients co-infected with HIV (PHOTON-1) 21st Conference on Retroviruses and Opportunistic Infections, March 3-6, 2014, Boston MA.

66. Lawitz E, Poordad F, Brainard DM, et al. Sofosbuvir with peginterferon-ribavirin for 12 weeks in previously treated patients with hepatitis C genotype 2 or 3 and cirrhosis. Hepatology 2015;61:769-775.

67. Gane E, Hyland R, An D, et al. Ledipasvir/sofosbuvir fixed dose combination is safe and effective in difficult-to-treat populations including GT 3 patients, decompensated GT 1 patients, and GT 1 patients with prior sofosbuvir experience. 49th Annual Meeting of the European Association for the Study of the Liver, April 9-13, 2014, London, UK.

68. Gane E, Hyland R, An D, et al. High efficacy of LDV/SOF regimens for 12 weeks for patients with HCV genotype 3 or 6 infection. American Association for the Study of Liver Diseases, 2014, Boston, MA.

69. Nelson DR, Cooper JN, Lalezari J, et al. All-oral 12-week combination treatmentwith daclatasvir and sofosbuvir in patients infected with HCV genotype 3: ALLY-3 phase 3 study. American Association for the Study of Liver Diseases, November 9-13, 2014, Boston, MA.

70. Pol S, Reddy R, Baykal T, et al. Interferon-free regimens of ombitasvir and ABT-450/r with or without ribavirin in patients with $\mathrm{HCV}$ genotype 4 infection: PERAL-I study results. American Association for the Study of Liver Diseases, 2014, Boston, MA.

71. Kapoor R, Kohli A, Sidharthan S, et al. Treatment of hepatitis $\mathrm{C}$ genotype 4 with ledipasvir and sofosbuvir for 12 weeks: Tesults fo the SYNDERGY trial. American Association for the Study of Liver Diseases, November 9-13, 2014, Boston, MA.

72. Ruane PJ, Ain D, Stryker R, et al. Sofosbuvir plus ribavirin for the treatment of chronic genotype 4 hepatitis $\mathrm{C}$ virus infection in patients of Egyptian ancestry. J Hepatol 2014; SO168-8278.

73. Wehmeyer MH, Eissing F, Jordan S, et al. Safety and efficacy of protease inhibitor based combination therapy in a single-center "real-life" cohort of 110 patients with chronic hepatitis C genotype 1 infection. BMC Gastroenterol 2014;14:87.

74. Mandorfer M, Payer BA, Niederecker A, et al. Therapeutic potential of and treatment with boceprevir/telaprevir-based triple-therapy in HIV/chronic hepatitis C co-infected patients in a real-world setting. AIDS Patient Care STDs 2014;28:221-227.

75. Dieterich D, Bacon B, Flamm S, et al. Evaluation of sofosbuvir and simeprevir-based regimens in the TRIO network-Academic and community treatment of a real-world, heterogeneous population. American Association for the Study of Liver Diseases, November 9-13, 2014, Boston, MA.

76. Jensen DM, O'Leary J, Pockros P, et al. Safety and efficacy of sofosbuvir-containing regimens for hepatitis C: Realworld experience in a diverse, longitudinal observational cohort. American Association for the Study of Liver Diseases, November 9-13, 2014, Boston, MA.

77. Del Bello D, Bichoupan K, Yalamanchili R, et al. Realworld data on HIV positive patients with HCV genotype 1,2 and 3 on sofosbuvir-and simeprevir-containing regimens. American Association for the Study of Liver Diseases, November 9-13, 2014, Boston, MA.

Address correspondence to:

Mamta K. Jain, MD, MPH Department of Internal Medicine UT Southwestern Medical Center 5323 Harry Hines Blvd Dallas, TX 75390-9113

E-mail: mamta.jain@utsouthwestern.edu 